\title{
COMPUTED TOMOGRAPHY IMAGING IN PYELONEPHRITIS AND ITS COMPLICATIONS
}

\author{
Murali Belman'1, Mandapal Toka², Santosh Hedau
}

${ }_{1}^{1}$ Associate Professor, Department of Radiology and Imaging Sciences, Bhaskar Medical College and General Hospital, Yenkapally, Telangana, India.

2Professor, Department of Radiology and Imaging Sciences, Bhaskar Medical College and General Hospital, Yenkapally, Telangana, India.

${ }^{3}$ Associate Professor, Department of Nephrology, Bhaskar Medical College and General Hospital, Yenkapally, Telangana, India.

\section{BACKGROUND}

ABSTRACT

Acute pyelonephritis (APN) is a common disease which rarely evolves into abscesses. Urinary tract infections are the common urologic disease and the estimated annual incidence of pyelonephritis is 10.5 million to 25.9 million cases globally. In general, routine CT imaging is not required for diagnosis and treatment of uncomplicated cases in adult patients. It is of value in establishing the diagnosis in equivocal cases, in evaluating high-risk patients, and in determining the extent of disease and reveal complications such as abscess or obstruction.

\section{METHODS}

This is a prospective study conducted over a period of 24 months from 2015 through 2017 . We collected clinical, biochemical and radiological data of patients hospitalized with a diagnosis of APN.

\section{RESULTS}

We identified the CT signs of acute pyelonephritis at different stages of the process. The typical CT-signs of AP were enlargement of the kidney with its thickened parenchyma and an impairment of corticomedullary differentiation. The highest incidence of acute pyelonephritis in our study was found in 41-50 years' age group accounting for $32 \%$ of cases and it was the least in the age group of 31-40 years constituting $0 \%$. Out of total patients, $92 \%$ had unilateral pyelonephritis, while $8 \%$ had bilateral pyelonephritis, right kidney was involved in $48 \%$ of cases and left kidney was involved in $52 \%$ of cases. These finding correlated with previous studies wherein the left kidney involvement in pyelonephritis was marginally higher when compared to the right renal involvement.

\section{CONCLUSIONS}

CT is particularly useful for evaluating the complications of pyelonephritis such as abscesses and emphysematous pyelonephritis. It helps in defining the extent and severity of these complications. CT plays a critical role in the management of these complications, especially in critically ill patients and reducing the morbidity and mortality. Guided procedures are recommended for the drainage of collections or abscesses.

\section{KEY WORDS}

CT, Acute Pyelonephritis, Focal Nephritis, Renal Abscess, Urinary Tract Infection

HOW TO CITE THIS ARTICLE: Belman M, Toka M, Hedau S. Computed tomography imaging in pyelonephritis and its complications. J. Evolution Med. Dent. Sci. 2019;8(28):2223-2229, DOI: 10.14260/jemds/2019/487

\section{BACKGROUND}

Pyelonephritis is classically defined as an infection of the upper urinary tract involving the renal parenchyma and pelvis. It normally occurs as secondary to a bacterial infection in the lower urinary tract usually involving the bladder or urethra. This infection then ascends to involve the kidneys to cause acute pyelonephritis.(1) The natural flow of urine in the urinary tract is thought to prevent the build-up of pathogenic bacteria. Hence, any obstruction can cause stasis of urine and create conditions suitable for infection.

'Financial or Other Competing Interest': None.

Submission 09-03-2019, Peer Review 25-06-2019,

Acceptance 02-07-2019, Published 15-07-2019.

Corresponding Author:

Dr. Murali Belman,

Plot 702- A, Road No. 36,

Jubilee Hills,

Hyderabad

Telangana, India

E-mail: drbelmanmurali@gmail.com

DOI: $10.14260 /$ jemds $/ 2019 / 487$

\section{(c) $(1)$}

There are a number of conditions that can interrupt the flow of urine and cause this infection to progress to pyelonephritis, such as ureteric calculi or obstruction secondary to prostatic hypertrophy in older males. Vesicoureteral reflux presents in approximately $10 \%$ of children and is found to be the cause of recurrent urinary tract infections in children. Females are predisposed to urinary tract infections, in general, due to their short urethra. The most common pathogen responsible found in such cases of ascending infection are Escherichia coli, Enterococci and rarely Klebsiella pneumonia. $(2,3)$

Infection may also spread to the kidney by the hematogenous route and in such cases of hematogenous seeding as a result of of a septic localisation in a patient with septicaemia, the most common pathogens involved are Staphylococci and Escherichia coli. The estimated annual incidence of pyelonephritis is 10.5 million to 25.9 million cases globally.(4)

Acute pyelonephritis is suggested by the constellation of fever, flank pain (typically unilateral), nausea and vomiting, and costovertebral angle tenderness.(5) Complaints typical of lower urinary tract infections such as dysuria and urinary 
frequency are variably present. The severity of symptoms ranges from a mild pyrexia illness to life-threatening sepsis.(6) Laboratory findings may include pyuria, leucocyte casts, bacteriuria and a positive urine culture. Blood tests may show leukocytosis with a neutrophilic shift, elevated erythrocyte sedimentation rate, elevated C-reactive protein levels, and occasionally positive blood cultures that grow the same organism as cultured from the urine.(7)

\section{Imaging Modalities}

Imaging is not routinely indicated in urinary tract infections, however with severe symptoms, high risk immunocompromised state, diabetic patients, and in those who are not responding to intravenous antibiotic therapy within 72 hours it becomes necessary. Plain radiography may provide evidence of gas in the renal area in emphysematous pyelonephritis or abscess and the typical mass like calcification in end stage renal tuberculosis (Putty kidney). Ultrasound (US) is the initial screening modality and is used for guiding interventions as well. It was reported that only around $20-25 \%$ of patients with acute pyelonephritis showed abnormalities on ultrasonography.(8,9) The role of intravenous urography (IVU) has diminished lately, however it still remains the best modality to diagnose calyceal irregularity of early tuberculosis, papillary necrosis and to evaluate congenital anomalies. Computed Tomography (CT) is considered the imaging modality of choice in the evaluation of patients with pyelonephritis and its complications.

Magnetic resonance imaging (MRI) is indicated in pregnancy and patients with contraindication to iodinated contrast such as transplant recipients. Diffusion weighted MRI (DW-MRI) has been applied to differentiate hydronephrosis from pyonephrosis as well as to detect infected cysts and tumours.

\section{Aims of The Study}

The aim of this research is to describe and analyse the role of CT in evaluation of acute pyelonephritis and its complications.

1. To identify and describe the Computed Tomography imaging appearances of pyelonephritis

2. To compare the Computed Tomography features of pyelonephritis with the clinical findings.

3. To identify and describe the Computed Tomography findings of complications associated with acute pyelonephritis and the extent of involvement, for planning of the management.

\section{METHODS}

This is a prospective study over a period of 2 years (2015 2017). A total of 50 patients were analysed retrospectively at the department of Radio diagnosis, Bhaskar General Hospital, Moinabad, Hyderabad.

All patients who are referred to the department of Radiodiagnosis for Computed Tomography (CT) scan with clinical suspicion/ diagnosis of acute pyelonephritis were consecutively inducted into the study after receiving informed consent.

In this study, the diagnosis of PN was based on clinical and radiological criteria as explained below.

\begin{tabular}{|c|c|c|}
\hline & No. of Cases & Extent of Disease \\
\hline Class 1 & 5 & Collecting System \\
\hline Class 2 & 3 & Renal Parenchyma \\
\hline Class 3 & 1 & Perirenal Extension \\
\hline Class 4 & 1 & Bilateral \\
\hline \multicolumn{3}{|c|}{ Table 1. Distribution of Cases of Emphysematous Pyelonephritis by } \\
Class
\end{tabular}
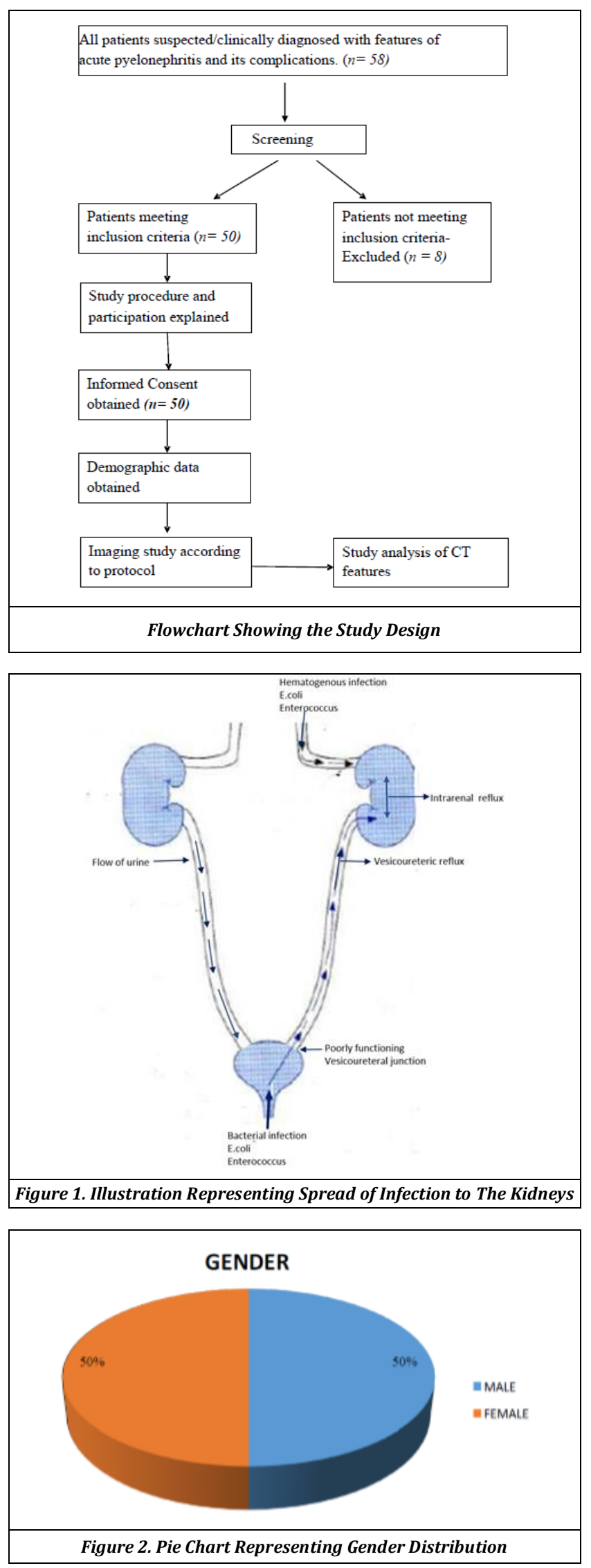

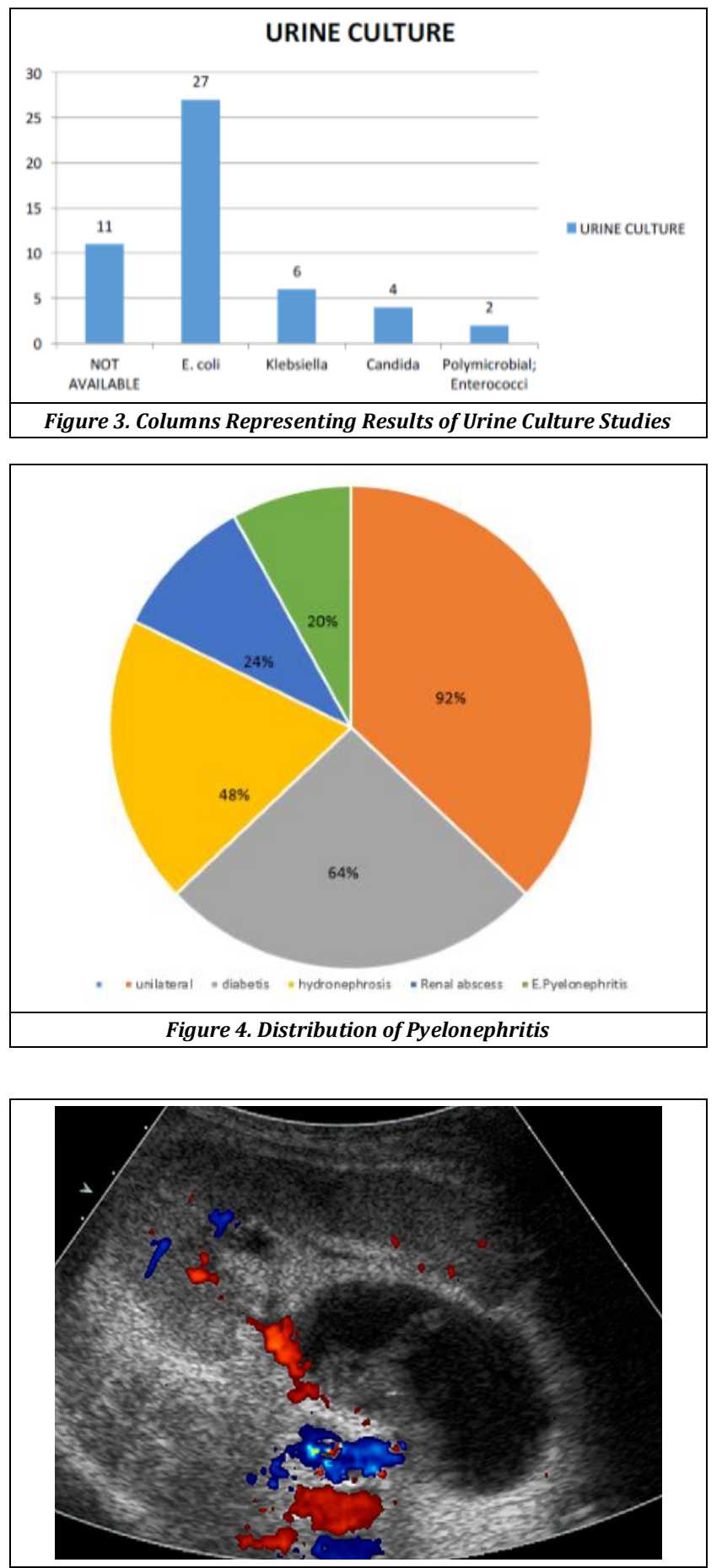

Figure 5. Ultrasound Image Showing Dilated Pelvis with Internal Echogenic Material Suggestive of Pyonephrosis

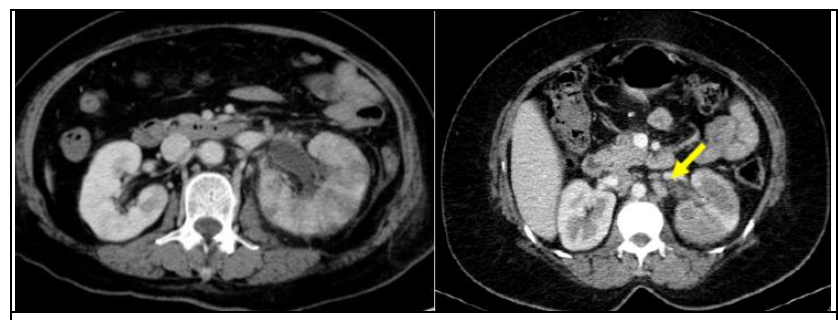

Figure 6. Axial CECT Images $(A, B)$ Shows Features of Acute Pyelonephritis of Left Kidney: Renal Swelling, Heterogenous Hypoenhancing Renal Parenchyma, Perinephric Fat Stranding, Thickening of Perirenal Fasciae, Hydronephrosis and Urothelial Thickening and Enhancement. An Enlarged Paraaortic Lymph Node (Yellow Arrow) at The Level of Left Renal Hilum is Noted (B)

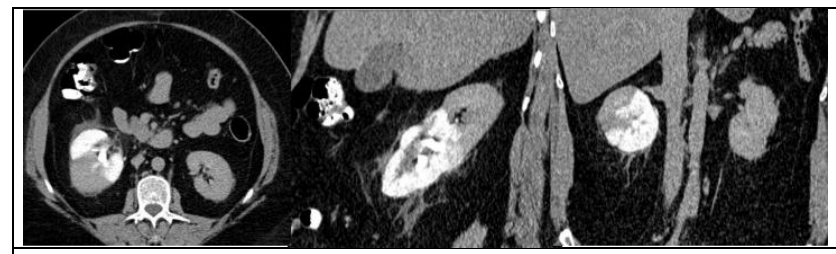

Figure 7. Axial, Sagittal, Coronal CECT Image Shows Features of Acute Pyelonephritis of Right Kidney: Renal Swelling, Focal Hypo Enhancing Renal Parenchyma, Perinephric Fat Stranding

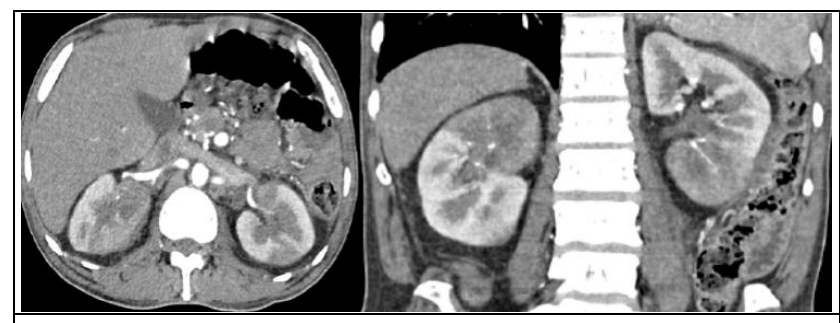

Figure 8. Axial and Coronal CECT Abdomen of a 45-Year-Old Male Patient Shows Mildly Enlarged Bilateral Kidneys with Focal WedgeShaped Hypo Enhancing Areas with Striated Pattern in Both Kidneys. There is Bilateral Thin Rim of Perinephric Fluid Collection. Features Suggestive of Bilateral Acute Pyelonephritis

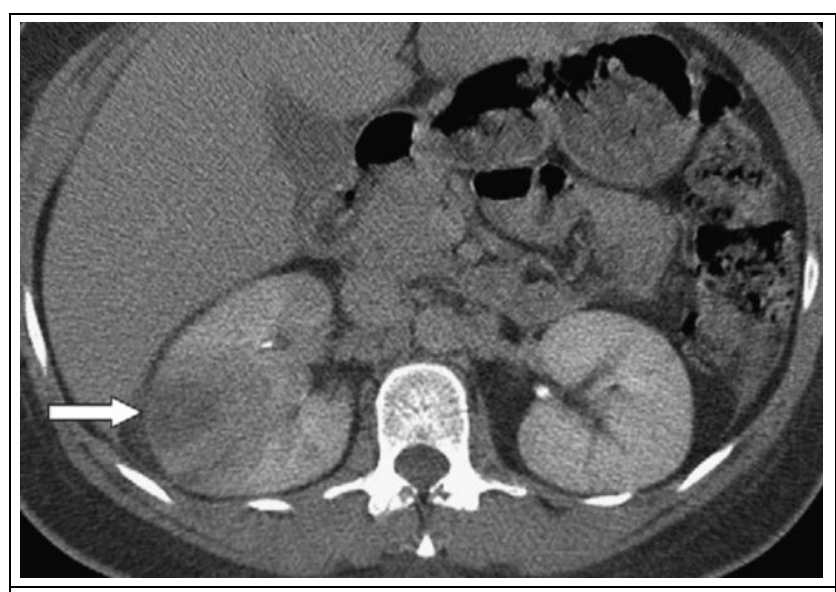

Figure 9. Axial CECT Image Shows a FOCAL Rounded Area of Decreased Attenuation with Peripheral Rim Enhancement, Suggestive of Renal Abscess

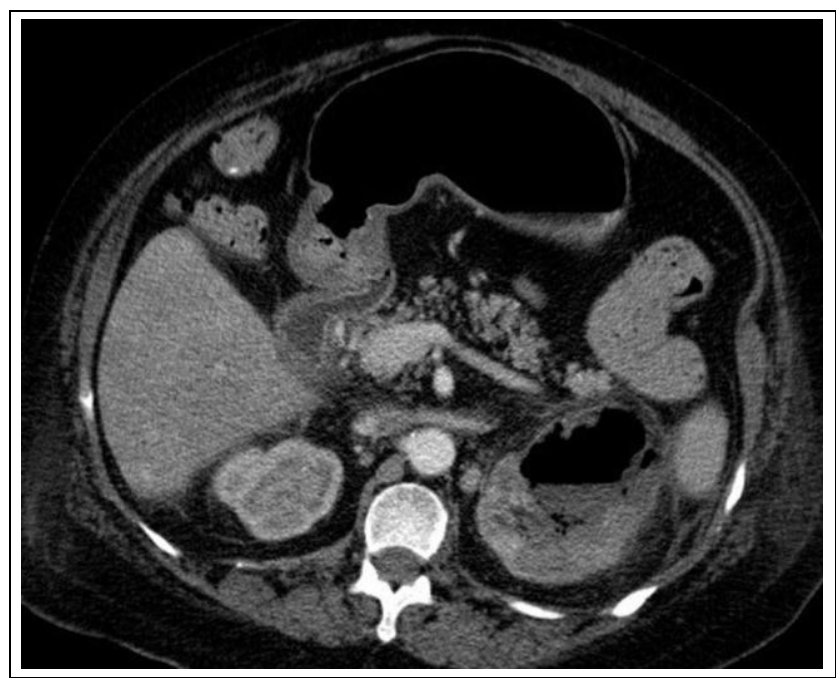

Figure 10. Axial CECT Image Shows Emphysematous Pyelonephritis of Left Kidney 


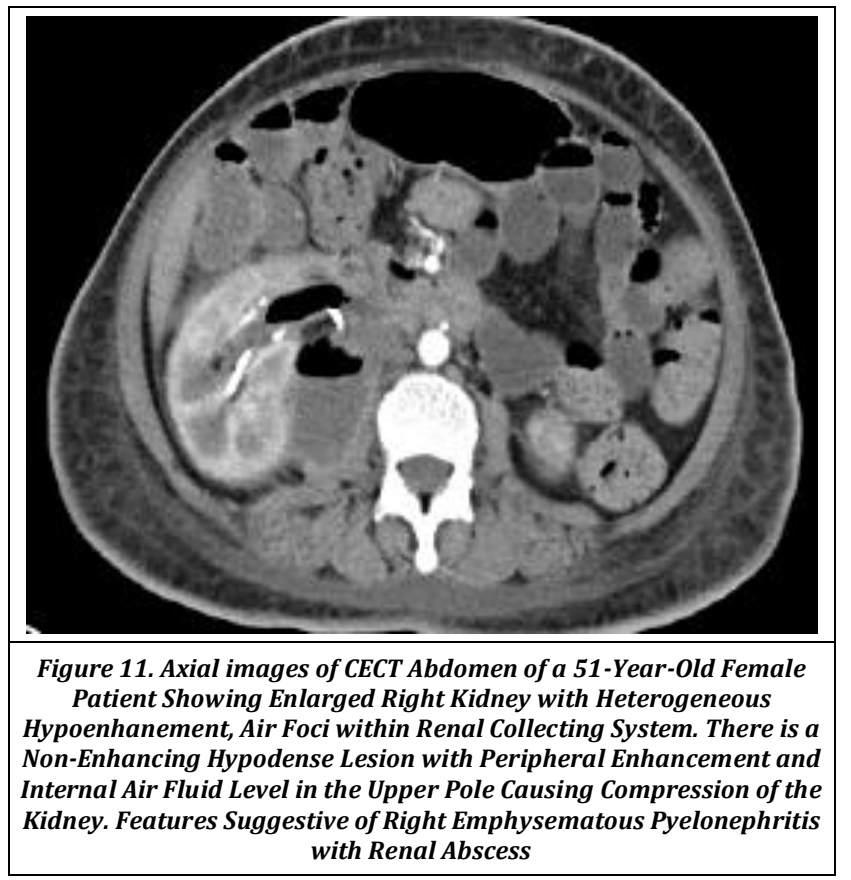

Clinical criteria were the presence of classical triad of PN i.e., fever with chills and rigor; presence of flank pain and pyuria.

Radiological diagnosis of PN was made when CT abdomen showed evidence of infection of renal parenchyma, in the form of bulky kidney, compression of renal sinus, perinephric fat stranding, abscess and emphysematous changes.

\section{Departments Involved}

1. Department of Radiodiagnosis.

2. Department of Nephrology.

3. Department of Microbiology.

\section{Inclusion Criteria}

The study included -

1. Patients with clinical features such as fever with chills, flank pain, nausea, vomiting, costovertebral angle tenderness.

2. Patients with laboratory findings such as pyuria, bacteriuria, leucocytosis, and positive urine culture.

3. Patients who needed computed tomography scan as a further investigation to rule out complications.

\section{Exclusion Criteria}

The study excluded the patients -

1. With history of chronic kidney disease whose serum creatinine values more than $1.2 \mathrm{md} / \mathrm{dl}$.

2. In whom contrast medium was contraindicated such as those who were allergic to the contrast medium.

3. Pregnant females.

4. Who didn't give consent for the study.

Imaging was performed on the Toshiba Alexion CT scan machine and sections from above the level of Diaphragm to Pubic symphysis. Plain study was first performed and Intravenous contrast at the rate of $4 \mathrm{ml} / \mathrm{sec}$ was injected to have the Corticomedullary (20-45 sec.) and Nephrogram (60$100 \mathrm{sec}$ ) phases. Delayed (Excretory/Urogram) phase (5-15 min.) were performed as needed.
The images were analysed by the research person/radiologist blinded to the clinical details and laboratory details.

Unenhanced CT was first used to identify calculi, haemorrhage, renal enlargement, inflammatory masses, wall calcifications and dilatation of collecting system. Calculi are seen as hyperdense foci of calcific densities. Once calculi were detected; number, size and location of the calculi were recorded. Presence or absence of renal enlargement, attenuation of the renal parenchyma, dilation of pelvicalyceal system and/or ureter were recorded. If the collecting system was dilated, the source of obstruction in the pelvicalyceal system and/or ureter as to the presence or absence of any calculi or soft tissue density lesions within the collecting system were evaluated.

Contrast enhanced CT was then used to detect wedge shaped Hypodensities, intrarenal abscesses and alternate striations of hypodensities. Other findings such as renal enlargement, perinephric fat stranding and thickening of Gerota's fascia were also evaluated on both plain and contrast enhanced CT scans. Presence of complications such as renal and/or perirenal abscesses, and the presence of gas were also identified. If abscesses were identified, then the number, size, location, internal characteristics, enhancement pattern, presence of air-fluid level or air foci within and around the abscesses were recorded. If air foci were identified, then the location, extent and spread of the air foci are recorded.

Pyelonephritis was classified into a) unilateral (right/left kidney or bilateral, b) focal or diffuse, c) with or without nephromegaly, and d) uncomplicated or complicated, based on different age groups and gender.

\section{Reference Standard}

The reference standard was clinical, urological and laboratory correlation.

\section{Statistical Analysis}

Descriptive statistics with percentages and proportions of the CT features of acute pyelonephritis and its associated complications were generated for the study patients according to-

- $\quad$ Age and sex.

- Gender.

- Presenting complaints.

- Diabetes mellitus.

- Urine examination.

- Urine culture.

- Unilateral/ bilateral involvement of kidneys.

- If unilateral, right or left kidney.

- Renal enlargement/ Bulky kidneys.

- Perinephric fat stranding/ thickening of perirenal fasciae or lateroconal fascia.

- Calculi.

- Dilation of pelvicalyceal system.

- Presence of focal or diffuse hypo enhancing areas within renal parenchyma.

- Paraaortic lymph nodes at the level of renal hila.

- Renal/ Perirenal abscesses.

- Air foci within renal parenchyma, collecting system or in the perirenal area - Emphysematous pyelonephritis. 


\section{RESULTS}

Fifty patients were evaluated, whose age group ranged from 13 to 80 years, out of which 25 (50\%) were males and 25 $(50 \%)$ were females. The highest incidence of acute pyelonephritis in the study was found in 41-50 years' age group accounting for $32 \%$ of cases and least was seen in age group of 31-40 years constituting $0 \%$.

This differed from the literature wherein acute pyelonephritis was more common in males than in females due to increased incidence of obstructive pathologies including benign prostatic hyperplasia.

Fever with chills and flank pain were the most common presenting complaints. Other symptoms included nausea and vomiting and urinary symptoms such as dysuria. Unilateral pyelonephritis was seen in $92 \%$, while 4 cases (8\%) had bilateral pyelonephritis.

In this study, 32 patients (64\%) were found to be diabetic and 18 patients (36\%) were non diabetic. As was previously described by Yadla $\mathrm{M}$ et al, our findings also coincided with the fact that the incidence of pyelonephritis and its complication was almost twice in diabetics compared to nondiabetics. Hence, proper control of diabetes is essential to reduce the morbidity and mortality associated with pyelonephritis.

This study found that all patients with clinically suspected pyelonephritis had pyuria that is above the normal threshold. Out of the 39 cases with urine culture results, 27 cases (69\%) had Escherichia coli infection. 6 cases (15\%) had urine cultures positive for Klebsiella organisms. All these cases were diabetic and were associated with complications of pyelonephritis. These findings reiterate the results from literature that showed Klebsiella infection is more common in diabetics and is associated with increased rate of pyelonephritis associated complications such emphysematous pyelonephritis. Of the remaining 6 cases, 4 (10\%) cases showed Candida positive 61 cultures and 2 (5\%) cases showed polymicrobial growth with predominant Enterococcus infection. The findings in this study were similar to the results obtained by Bailey BL et al who studied the urine culture results of upper and lower urinary tract infections.

Enlarged kidneys or bulky kidneys are found in 40 (80\%) cases with pyelonephritis, while normal sized kidneys were found in $10(20 \%)$ cases. Perinephric fat stranding or thickening of the anterior perirenal fascia (Gerota's fascia), posterior perirenal fascia (Zuckerkandl's fascia) and lateroconal fascia was found in $47(94 \%)$ cases, while these features were absent in $3(6 \%)$ cases.

Hydronephrosis was seen in 24 (48\%) cases, while in 26 $(52 \%)$ cases, the pelvicalyceal system and collecting system were normal. The dilated pelvicalyceal system was either due to calculi $(8 \%)$ or due to sloughed off papillae or inflammatory debris which was seen as hyperdense soft tissue density within the collecting system or ureters.

Enlarged/prominent paraaortic or paracaval or aortocaval lymph nodes at the level of renal hila were assessed and found in 25 (50\%) cases. and more commonly seen in complicated cases of pyelonephritis.

Hypo-enhancing or non-enhancing areas of renal parenchyma were found in 39 cases (78\%) out of the total 50 cases. According to several studies in the literature, this is the most definitive sign in the diagnosis of acute pyelonephritis.
These hypo-enhancing areas represent early evolving abscesses and micro abscesses and if left untreated, develop into renal abscesses. In a few cases, these hypo-enhancing areas gave rise to a striated nephrogram appearance.

The two common complications of acute pyelonephritis that were observed in this study are renal and perirenal abscesses, and emphysematous pyelonephritis. Of the 50 cases, 3 patients had both complications simultaneously i.e., they had both renal/perirenal abscess formation and emphysematous pyelonephritis.

Out of the 50 cases evaluated, 12 cases (24\%) showed abscess formation. Intrarenal abscesses were found in 9 cases out of the 12 patients, while the remaining 3 had perirenal abscesses. One patient out of the 3 had a perirenal abscess extending along the psoas muscle into the pelvis.

10 cases $(20 \%)$ out of the total 50 cases evaluated, showed features of emphysematous pyelonephritis, a severe necrotizing complication of acute pyelonephritis. All the 10 patients were diabetics. There was $100 \%$ association between emphysematous pyelonephritis and diabetes mellitus in this study.) According to Huang-Tseng classification, in this study, the distribution of cases of emphysematous pyelonephritis is as follows:

4 of the 10 cases of emphysematous pyelonephritis showed Klebsiella as the infective organism. 4 of the 10 cases had to undergo nephrectomy, while 6 of them were managed with nephrostomy tube.

\section{DISCUSSION}

CT imaging is a non-invasive, multiplanar and highly accurate method with better inherent contrast that demonstrates the features of acute pyelonephritis and its complications accurately. This was a prospective observational study done aimed at studying the CT findings of acute pyelonephritis and its complications. In the present study for the same, we evaluated 50 patients.

\section{Acute Pyelonephritis}

Acute pyelonephritis is defined as an infection of the upper urinary tract, specifically the renal parenchyma and renal pelvis. Non-enhanced CT may appear normal or show subtle changes in the presence of pyelonephritis. Therefore, contrast enhanced scans should be performed following the unenhanced scan.

The recommended phases of CT scan for evaluating renal infections are a non-contrast scan, Corticomedullary (20-45 sec.), Nephrogram (60-100 sec) phases and delayed (Excretory/Urogram) phase (5-15 $\mathrm{min}$.) if there is obstruction.

Acute bacterial nephritis most commonly manifests as one or more wedge-shaped areas of decreased attenuation or a hypodense mass.

These areas of focal hypoattenuation are also seen in tumours, renal infarcts, scarring and in medullary sponge kidney. However, in these scenarios, the hypoattenuating areas persist even after antibiotic therapy, whereas in acute pyelonephritis, the areas show resolution.

Another appearance that may be seen is the striated nephrogram, shows discrete rays of alternating hypoattenuation and hyper attenuation radiating from the papilla to the cortex. This appearance occurs due to the decreased flow of contrast due to stasis and eventual 
hyperconcentration in the infected tubules. Striated nephrogram is not specific to pyelonephritis and is also seen in some other conditions like renal vein thrombosis, ureteric obstruction and renal contusion. Most patients with acute pyelonephritis show bulky or enlarged kidneys, poor enhancement of renal parenchyma, absent excretion of contrast, perinephric fat stranding and thickening of the pararenal fasciae. Presence of perinephric stranding is not useful in diagnosis as it is not a specific sign for acute pyelonephritis. It is seen in other conditions such as previous infection, obstructive uropathy, following trauma or renal interventions and in chronic pyelonephritis.

\section{Complicated Pyelonephritis/Renal and Perirenal Abscesses}

In a few cases of acute pyelonephritis, severe vasospasm and inflammation may result in liquefactive necrosis and abscess formation. Abscess cavities may be either intraparenchymal or extraparenchymal, and a renal abscess should be suspected when appropriate therapy does not lead to clinical response. $75 \%$ of all renal abscesses occur in diabetic patients as they are predisposed to abscess formation.(10)

An abscess usually appears as a well-defined collection of low attenuation with a thick, irregular wall that enhances on contrast enhanced CT. A halo of diminished enhancement may surround the abscess during the nephrographic phase, which shows enhancement on delayed imaging.(11)

A perinephric abscess may result from rupture of a renal abscess into the perirenal space or from extension of inflammatory disease outside the Gerota fascia. Diabetic patients with calculi and patients with septic emboli are prone to this complication. Perinephric abscesses are usually limited to perirenal space but may involve the psoas muscle and extend to the pelvis and groin

\section{Emphysematous Pyelonephritis}

Emphysematous pyelonephritis is a severe, necrotising form of acute bacterial pyelonephritis in which renal parenchyma infection with gas-forming bacteria occurs. $90 \%$ of patients who develop this condition have a history of poorly controlled diabetes mellitus.(12) Obstruction of the collecting system may be a cause in nondiabetic patients. Emphysematous pyelonephritis is nearly twice as common in females in whom it is usually unilateral.(13) Patients present with a palpable flank mass and carries a high mortality rate of $50 \%$ due to rapid progression to septic shock. The most common organism involved is Escherichia coli (70\%), followed by Klebsiella and Candida.

CT scanning is the modality of choice in the evaluation of these patients. It confirms the presence of gas and also defines the extent of disease. Parenchymal gas characteristically appears as multiple small air bubbles or linear streaks radiating from the renal papillae, illustrating the gas dissecting along the interstitial planes.

Two types of classification systems are present based on CT findings. The more common classification divides emphysematous pyelonephritis into 2 types.

Type 1 is characterised by parenchymal destruction, with streaky or mottled gas collections and the absence of fluid collections. Type 2 is characterised by bubbly or loculated gas collections within the parenchyma or collecting system, with renal or perirenal fluid collections.(14)
A second CT based classification described by HuangTseng et al,(15) differentiates emphysematous pyelonephritis into 4 types as follows-

- Class 1: gas in collecting system only (Emphysematous pyelitis).

- Class 2: gas in renal parenchyma only (Without extrarenal extension).

- Class 3: gas in renal parenchyma with extrarenal extension.

- Class 3a: extension of gas or abscess to perinephric space.

- Class 3b: extension of gas or abscess to pararenal space.

- Class 4: bilateral emphysematous pyelonephritis or solitary kidney with emphysematous pyelonephritis.

The line of management includes aggressive medical treatment along with percutaneous drainage and partial or complete nephrectomy, thereby reducing the mortality associated with this condition.

\section{Pyonephrosis}

Pyonephrosis is an infected and obstructed collecting system, which frequently is enlarged. Pyonephrosis should be suspected in any patient with a known urinary tract obstruction and accompanying fever and flank pain. CT demonstrates thickening of the renal pelvic wall $(>2 \mathrm{~mm}$ ), parenchymal or perinephric inflammatory changes, dilatation and obstruction of the collecting system, higher than usual attenuation values of the fluid within the renal collecting system, and layering of contrast material above and anterior to the purulent fluid on excretory studies.(16)

\section{CONCLUSIONS}

CT is the imaging modality of choice for the diagnosis of acute pyelonephritis and its associated complications due to its wide availability and accurate depiction of the features, extent and severity of the disease. The incidence of pyelonephritis and its complications had a strong association with the presence of diabetes mellitus in patients and as such careful evaluation of these patients is required to reduce the risk of complications in such patients. CT plays a critical role in patient management by suggesting the correct diagnosis based on characteristic imaging findings, identifying the complications especially in critically ill patients and reducing the morbidity and mortality by immediate percutaneous nephrostomy or nephrectomy in cases of complications of pyelonephritis.

\section{REFERENCES}

[1] Stamm WE, Hooton TM, Johnson JR, et al. Urinary tract infections: from pathogenesis to treatment. J Infect Dis 1989;159(3):400-6.

[2] Colgan R, Williams M, Johnson JR. Diagnosis and treatment of acute pyelonephritis in women. Am Fam Physician 2011;84(5):519-26.

[3] Ramakrishnan K, Scheid DC. Diagnosis and management of acute pyelonephritis in adults. Am Fam Physician 2005;71(5):933-42.

[4] Czaja CA, Scholes D, Hooton TM, et al. Populationbased epidemiologic analysis of acute pyelonephritis. Clin Infect Dis 2007;45(3):273-80. 
[5] Talner LB, Davidson AJ, Lebowitz RL, et al. Acute pyelonephritis: can we agree on terminology? Radiology 1994;192(2):297-305.

[6] Keenan DB, O'Rourke DM, Courtney AE. Pyelonephritis can lead to life-threatening complications. Practitioner 2017;261(1801):17-20.

[7] Hooton TM. Clinical practice. Uncomplicated urinary tract infection. N Engl J Med 2012;366(11):1028-37.

[8] Vourganti S, Agarwal PK, Bodner DR, et al. Ultrasonographic evaluation of renal infections. Radiol Clin North Am 2006;44(6):763-75.

[9] June CH, Browning MD, Smith LP, et al. Ultrasonography and computed tomography in severe urinary tract infection. Arch Intern Med 1985;145(5):841-5.

[10] Thornbury JR. Acute renal infections. Urol Radiol 1991;12(4):209-13.

[11] Deyoe LA, Cronan JJ, Lambiase RE, et al. Percutaneous drainage of renal and perirenal abscesses: results in 30 patients. American Journal of Roentgenology 1990;155(1):81-3.
[12] Evanoff GV, Thompson CS, Foley R, et al. Spectrum of gas within the kidney: emphysematous pyelonephritis and emphysematous pyelitis. The American Journal of Medicine 1987;83(1):149-54.

[13] Grozel F, Berthezene Y, Guerin C, et al. Bilateral emphysematous pyelonephritis resolving to medical therapy: demonstration by US and CT. European Radiology 1997;7(6):844-6.

[14] Wan YL, Lee TY, Bullard MJ, et al. Acute gas-producing bacterial renal infection: correlation between imaging findings and clinical outcome. Radiology 1996;198(2):433-8.

[15] Huang JJ, Tseng CC. Emphysematous pyelonephritis: clinicoradiological classification, management, prognosis and pathogenesis. Archives of Internal Medicine 2000;160(6):797-805.

[16] Fultz PJ, Hampton WR, Totterman SM. Computed tomography of pyonephrosis. Abdominal Imaging 1993;18(1):82-7. 